\title{
Feststoffmanagement bei Wasserkraftanlagen in Österreich
}

\author{
Norbert Bock · Gottfried Gökler · Robert Reindl · Josef Reingruber · Roland Schmalfuß · Hannes Badura · \\ Gerd Frik · Ines Leobner · Josef Lettner · Martin Scharsching · Reinhard Spreitzer · Marie-Theres Thöni
}

Online publiziert: 5. Februar 2019

(C) Der/die Autor(en) 2019

Zusammenfassung Die Veränderung des Feststoffhaushalts durch die Wasserkraftnutzung ist aufgrund natürlicher, physikalischer Zusammenhänge unvermeidlich, kann jedoch durch konstruktive Maßnahmen bei der Kraftwerksplanung sowie durch an die natürlichen Verhältnisse angepasste Betriebsführung reduziert werden. Feststoffmanagementmaßnahmen wie Stauraum- bzw. Speicherspülungen und Kompensationsbaggerungen sind daher bei vielen Kraftwerksanlagen heute unumgänglich. Neuanlagen müssen bereits in der Konzeption ein nachhaltiges Feststoffbewirtschaftungskonzept beinhalten. Bei Bestandsanlagen ist in einer Einzelfallbetrachtung eine nachhaltige Lösung mit der geringstmöglichen Beeinflussung der Betriebsweise anzustreben. Dabei sollte die gesamthafte Betrachtung von Feststoffmanagementkonzepten immer flussgebietsbezogen und sektorenübergreifend sein und sämtliche Stakeholder einbeziehen.

Anhand von Fallbeispielen bei österreichischen Wasserkraftanlagen werden

\section{N. Bock}

Wien Energie GmbH,

Thomas-Klestil-Platz 14, 1030 Wien,

Österreich

DI G. Gökler

Vorarlberger Illwerke AG,

Anton-Ammann-Straße 12, 6773 Vandans, Österreich

DI Dr. R. Reindl

TIWAG-Tiroler Wasserkraft AG,

Eduard-Wallnöfer-Platz 2, 6020 Innsbruck, Österreich

\section{J. Reingruber}

Salzburg AG für Energie, Verkehr und Telekommunikation,

Bayerhamerstraße 16, 5020 Salzburg, Österreich

DI Dr. R. Schmalfuß (殴 .

DI Dr. H. Badura • Mag. G. Frik VERBUND Hydro Power GmbH, Europaplatz 2, 1150 Wien, Österreich Roland.Schmalfuss@verbund.com aktuelle Anwendungen von Methoden des Feststoffmanagements beschrieben, die notwendigen Optimierungen erläutert und Anforderungen an die Forschung aufgezeigt. Insbesondere in Hinblick auf Prozessverständnis und Maßnahmenkonzeption besteht weiterhin Forschungsbedarf, der für die österreichischen Wasserkraftbetreiber wesentlicher Anlass für die Unterstützung des von der Christian Doppler Forschungsgesellschaft 2017 eingerichteten Labors für „Sedimentforschung und -management" ist.

Schlüsselwörter Wasserkraft .

Sediment · Schwebstoff - Geschiebe .

Feststoffmanagement .

Stauraumspülung $\cdot$ Speicherverlandung

\section{Sediment Management at Hydro Power Plants in Austria}

Abstract Hydropower inevitably changes the sediment balance due to natural and physical relations. The change, however, can be reduced by construc-

DI Dr. I. Leobner

ÖBB-Infrastruktur AG,

Praterstern 3, 1020 Wien, Österreich

\section{J. Lettner}

Ennskraftwerke AG,

Resthofstraße 2, 4400 Steyr, Österreich

DI M. Scharsching

evn naturkraft Erzeugungsgesellschaft mbH, EVN-Platz 1, 2344 Maria

Enzersdorf, Österreich

DI R. Spreitzer

Energie AG Oberösterreich

Kraftwerke $\mathrm{GmbH}$,

Böhmerwaldstraße 3, 4020 Linz,

Österreich

DI M.-T. Thöni

Österreichs E-Wirtschaft,

Brahmsplatz 3, 1040 Wien, Österreich tive measures in power plant design as well as by an operational management that is adapted to the natural conditions. Therefore, for many hydropower plants the need for sediment management measures, such as reservoir flushing and compensational dredging, arises. The conceptual design of new plants must therefore already contain a sustainable sediment management concept. In the case of existing plants, a case-by-case analysis should be conducted to identify a sustainable solution with the least possible influence on the mode of operation. The overall consideration of sediment management concepts should always be river basinrelated and cross-sectoral and should involve all stakeholders.

On the basis of case studies of Austrian hydropower plants, current applications of sediment management methods, necessary optimization measures and research requirements are illustrated. Particularly with regard to process understanding and the conception of measures, there is still a need for research, which is an important reason for Austrian hydropower operators to support the Laboratory for "Sediment Research and Management", which was established by the Christian Doppler Research Association in 2017.

Keywords Hydropower - Sediment . Suspended load · Bed load · Sediment management - Reservoir flushing • Reservoir sedimentation

\section{Einleitung}

Die mit Errichtung und Betrieb von Wasserkraftanlagen einhergehenden Veränderungen der hydromorphologischen Verhältnisse eines Fließgewässers haben vielfach unvermeidliche Auswirkungen auf den Feststoffhaushalt des Gewässers. In den meisten Fällen werden Managementmaßnahmen erforderlich, um nachteilige Auswirkungen auf Dritte oder den gewässerökologischen Zustand zu vermeiden bzw. zu vermindern. 
In Umsetzung der Ziele der Wasserrahmenrichtlinie in Österreich sieht der Nationale Gewässerbewirtschaftungsplan (NGP) 2015 unter anderem die Erstellung von Feststoffmanagementkonzepten sowie die Untersuchung von Baumaßnahmen, Bauwerken und Betriebs- bzw. Managementweisen in Hinblick auf den Feststofftransport vor (BMLFUW 2015a). Zur Vorbereitung des NGP 2015 wurden im Rahmen des Projektes SED_AT (BMLFUW 2014) die relevanten Themen sowie der Forschungsund Managementbedarf in den gewässerrelevanten Sektoren erhoben und daraus integrativer Handlungsbedarf abgeleitet (Habersack et al. 2014).

Das von der Christian Doppler Forschungsgesellschaft unter Mitwirkung der österreichischen Wasserkraftbetreiber 2017 eingerichtete Labor für Sedimentforschung und -management (CD-Labor Sediment) unter der Leitung von Priv.-Doz. DI Dr. Christoph Hauer widmet sich der anwendungsorientierten Grundlagenforschung in diesem Fachbereich. Um ein erweitertes Prozessverständnis zu erzielen, ist eine Kombination aus Laborversuchen und Felduntersuchungen vorgesehen. Dabei werden die Grundlagen von Erosion, Transport, Sedimentation und Remobilisierung von Feststoffen erforscht. Die Forschungsansätze umfassen unterschiedliche Skalen, vom $\mu \mathrm{m}$ Bereich bis zur Untersuchung ganzer Einzugsgebiete und erfolgen in Kombination unterschiedlicher Methoden. Darauf aufbauend sind Erkenntnisse über Wechselwirkungen und Beeinflussungen zwischen Ökologie und Sedimenthaushalt und somit Grundlagen zur Entwicklung von neuen Monitoring- und Modellierungstechnologien $\mathrm{zu}$ erwarten. Hauer et al. (2018) liefert eine detaillierte Beschreibung der Ausgangsbasis und der Forschungsvorhaben.

Im Modul 1 werden Möglichkeiten zur optimierten ökonomischen, technischen bzw. ökologischen Nutzung der Wasserkraft auf wissenschaftlicher Basis behandelt. Der Verein für Ökologie und Umweltforschung (VOEU) koordiniert einzelne Forschungsprojekte der österreichischen Kraftwerksbetreiber und ist als Vertreter für die Wasserkrafterzeugung Wirtschaftspartner des Moduls 1. Zur Konkretisierung der im Rahmen des Projekts zu behandelnden Fragestellungen sowie zur zeitlichen und inhaltlichen Projektsteuerung wurde im Rahmen von Oesterreichs Ener- gie, welche als Interessenvertretung der österreichischen Elektrizitätswirtschaft die Position der Kraftwerksbetreiber vertritt, ein Fachbeirat eingesetzt, der sich aus Mitgliedern des CD-Labors und Vertretern der mitwirkenden Kraftwerksunternehmen zusammensetzt. Als Untersuchungsschwerpunkte werden von den Wasserkraftbetreibern folgende Themen gesehen:

- Bei Speicherkraftwerken steht die Verlandung infolge des Eintrags von Schwebstoffen und Geschiebe im Mittelpunkt. Neben dem Erhalt des Speichernutzvolumens ist auch die Erhaltung der Anlagensicherheit bezüglich der Betriebseinrichtungen ein zentrales Anliegen, das unter Berücksichtigung der naturräumlichen und gewässerökologischen Verhältnisse eine große Herausforderung darstellt und oft aufwendige Managementmaßnahmen erfordert.

- Der Schwebstoffeintrag in Stauräume ist auch bei Flusskraftwerken von großer Bedeutung; insbesondere, wenn die Verlandung von Seitenbereichen gewässerökologische Beeinträchtigungen bewirkt. Die Remobilisierung von Feinsedimentablagerungen im Zuge von Hochwasserereignissen kann zu lokal erhöhten Feinsedimentablagerungen in Überflutungsgebieten führen und damit Beeinträchtigungen für Landund Forstwirtschaft, aber auch von Siedlungsgebieten verursachen. Hier wird im Rahmen des CD-Labors eine Verbesserung des Prozessverständnisses angestrebt.

- Neben Schwebstoffen ist bei Flusskraftwerken auch der Geschiebehaushalt von Bedeutung. Teilweise wird durch konstruktive Ausgestaltung der Wehranlagen und geeignete Stauregelungen versucht, einen durchgängigen Geschiebetransport zu erhalten, was besonders bei Kraftwerksketten eine große Herausforderung darstellt. Wo dies aufgrund der baulichen und wasserwirtschaftlichen Rahmenbedingungen nicht möglich ist, muss in Übergangsbereichen mit Anlandungen oder Sohleintiefungen gerechnet werden, die aufwendige Geschiebebewirtschaftungsmaßnahmen erfordern können.

Im vorliegenden Beitrag werden diese Aspekte durch einige ausgewählte Fallbeispiele illustriert. Zuvor wird die Wirkung von Wasserkraftanlagen auf den
Feststoffhaushalt auf allgemeiner physikalischer Ebene betrachtet.

\section{Energieumwandlung im Fließgewässer oder an der Turbine}

In einem natürlichen Fließgewässer erfolgt die Umwandlung der potenziellen Energie des Wassers einerseits durch Reibung an der Gewässersohle, andererseits durch die Turbulenz der Strömung im Inneren des Wasserkörpers. Beide Prozesse stehen in engem Zusammenhang mit dem Feststofftransport:

- Die Sohlschubspannung, welche der fließende Wasserkörper auf die Stromsohle ausübt, treibt den Geschiebetransport auf oder nahe der Gewässersohle an.

- Die Turbulenz der Strömung wiederum ist erforderlich, um Schwebstoffe dauerhaft in Schwebe zu halten und damit Voraussetzung für deren Transport mit der fließenden Welle.

Effiziente Wasserkraftanlagen werden so konzipiert und angeordnet, dass ein großer Teil der potenziellen Energie des Wassers im Einflussbereich der Anlage nicht mehr in der Fließstrecke des Gewässers, sondern an der Turbine in elektrische Energie umgewandelt wird:

- Bei Flusskraftwerken werden Energieliniengefälle und Fließgeschwindigkeit im Stauraum durch Spiegelanhebung und damit einhergehende Vergrößerung des Abflussquerschnitts gegenüber der freien Fließstrecke stark verringert. Dies bewirkt im Stauraum in weiterer Folge eine deutliche Reduktion der Turbulenz der Strömung sowie der Sohlschubspannung und damit unvermeidlich auch eine Reduktion sowohl des Schwebstoff- als auch des Geschiebetransports.

- Bei Ausleitungskraftwerken wird zudem ein großer Teil des Gesamtabflusses über einen zumeist hydraulisch glatt ausgebildeten Triebwasserweg geführt, sodass in der frei fließenden Restwasserstrecke im Normalfall nur mehr ein deutlich reduzierter Durchfluss und dadurch eine verringerte Feststofftransportkapazität zur Verfügung steht.

Somit besteht bereits auf physikalischer Ebene ein grundsätzlicher Konflikt zwischen Wasserkraftnutzung und Feststofftransport. Die durch die Wasserkraftanlage genutzte Energie steht 
nicht mehr zum Antrieb des Feststofftransports zur Verfügung. Dieser fundamentale Konflikt wird jedoch durch folgende Aspekte gemildert:

- Aufgrund des stark nicht linearen Zusammenhangs zwischen Durchfluss und Feststofftransport erfolgt bei vielen Fließgewässern ein Großteil des Schwebstoff- und auch ein erheblicher Anteil des Geschiebetransports im Zuge von Hochwasserereignissen. Da der Stau von Flusskraftwerken bei Hochwasser oft gelegt werden muss bzw. bei Ausleitungskraftwerken ein großer Teil der Hochwasserfracht über die Restwasserstrecke abfließt, wird in diesen Phasen auch ein großer Anteil der potenziellen Energie in den Fließstrecken umgewandelt und steht damit wieder für den Antrieb des Feststofftransports zur Verfügung. Durch entsprechend angepasste Betriebsführung der Kraftwerksanlagen kann das Hochwasserereignis gezielt zur Spülung von Stauräumen und Restwasserstrecken genutzt werden. Andererseits kann die erhöhte Transportkapazität in den Restwasserstrecken auch zu ungewünschten Austragungseffekten führen.

- Der „Wirkungsgrad“ des Feststofftransports in natürlichen Fließgewässern dürfte im Allgemeinen gering sein; d.h., nur ein relativ kleiner Teil der umgewandelten potenziellen Energie wird tatsächlich für den Antrieb der Feststofftransportprozesse benötigt. Dieser hier nur qualitativ angesprochene Umstand bedarf noch fundierter wissenschaftlicher Untersuchung und Quantifizierung, er bildet jedoch die physikalische Grundlage für die bei manchen Wasserkraftanlagen mögliche Verbesserung der Feststoffdurchgängigkeit durch spezielle konstruktive Maßnahmen wie abgesenkte Wehrschwellen, Geschiebegassen und Bypassstollen.

\section{Alpine Speicher}

Bei Speicherkraftwerken stellen die Talsperrenanlagen wie Dämme und Staumauern zur Schaffung großer künstlicher Speicherseen eine abrupte und bedeutende Unterbrechung des natürlichen dynamischen Feststofftransports dar. Durch den Aufstau wird wie bei natürlichen Seen das gröbere Sediment in den meisten Fällen im hinteren Stauraum vollständig in Form eines
Deltas abgelagert, während die feineren Sedimente bis in den vordersten Teil des Stausees transportiert werden und sich dort vor Betriebseinläufe und Grundablass ablegen. Ein großer Teil der Schwebstoffe wird günstigerweise bereits mit dem Triebwasser weitertransportiert. Allerdings können sich diese bei langem Aufenthalt in strömungsberuhigten Bereichen des Stauraums auch endgültig und nachteilig für den Betrieb absetzen. Ebenso können fallweise Dichte- bzw. Trübeströme, die sich unter Umständen bereits bei kleineren Hochwasserereignissen in den Speichern ausbilden, Auswirkungen auf die Speicherverlandung haben. Bei vielen alpinen Speichern wird das Einzugsgebiet durch Bei- und Überleitungen vergrößert. Bei deren Einmündung, die meist nicht im Stauwurzelbereich liegt, können zwar keine Geschiebe, jedoch ebenfalls mehr Schwebstoffe in den Speicher transportiert werden.

Der unterbrochene Sedimenttransport führt einerseits zu kontinuierlicher Verlandung der Speicherräume und den damit einhergehenden betrieblichen Schwierigkeiten. Andererseits kann das Feststoffdefizit unterhalb der Talsperren bzw. Speicherentleerungen und Grundablassspülungen durch den kurzzeitig hohen Austrag von Feinsedimenten ökologische Probleme mit sich bringen.

Dabei kommt aus Sicht der Betreiber von Speicherkraftwerken folgenden Punkten wesentliche Bedeutung zu:

- Die vorrangige Aufgabe ist der dauerhaft sichere Betrieb eines Speichers in Hinblick auf die öffentliche Sicherheit und die Einhaltung der Bewilligungsvorschreibungen. Der Wasserkraftbetreiber muss die jederzeitige Betriebssicherheit der Betriebsorgane wie Einlauf und Grundablass gewährleisten und darauf achten, dass die statisch maximal zulässigen Verlandungshöhen an der Staumauer nicht überschritten werden. Ein (nachhaltiges) Sedimentmanagement erfordert, dass Verlandungen vor den Einlaufbereichen der Betriebsorgane derart hintanzuhalten sind, dass ein sicheres Öffnen und Schließen der Entlastungsorgane dauerhaft gegeben ist. Hier stellt z. B. das Nachrutschen bestehender Sedimentablagerungen bei Pegelabsenkungen im Zuge von Speicherentleerungen oder Spülvorgängen eine besondere Gefahr dar. Hinsichtlich des Ablaufs solcher Erosionsprozes- se sowie der Remobilisierung von Sedimenten im Stauraum besteht in weiten Bereichen auch zukünftig noch erheblicher Forschungsbedarf.

- Ziel der Stauraumbewirtschaftung ist, langfristig den Verlust von Nutzvolumen in Grenzen zu halten und einen optimalen Speicherbetrieb zu ermöglichen. Nachdem inzwischen bei vielen alpinen Speichern zufolge eines jahrzehntelangen Sedimenteintrags bereits eine weitgehende Totraumverlandung gegeben ist, können die ansonsten unabwendbaren betrieblichen Einschränkungen nur mit nachhaltigen Sedimentmanagementmaßnahmen hintangehalten werden. Dazu ist es primär erforderlich, die Eintrags- und Ablagerungsraten in die Speicher zu erheben und über die (technische) Lebensdauer darzustellen. Aufgrund teilweise fehlender Kenntnisse stellt der Klimawandel mit dem erwarteten Gletscherrückgang in diesem Zusammenhang eine große Unsicherheit dar. Generell ist mit der Verschiebung des Permafrosts in höhere Regionen von einer Erhöhung des Geschiebe- und Schwebstoffdargebotes auszugehen. Die wissenschaftliche Erarbeitung von Prognosewerten für die nächsten Jahrzehnte stellt für Wasserkraftbetreiber eine wesentliche Grundlage zur Abwägung und Erarbeitung nachhaltiger Konzepte dar. Derartige Randbedingungen beeinflussen Entscheidungen, ob eine Verlagerung in unproblematische Bereiche des Stauraums, eine Entnahme und Deponierung, gegebenenfalls eine Abkehr von Einleitungen bei sehr hoher Schwebstoffführung oder regelmäßige Spülungen die effizienteste Managementmaßnahme ist, wesentlich.

- Sedimenttransport in die Nähe der Betriebseinläufe bewirkt in der Folge einen starken Sedimenteinzug, sodass es durch Hydroabrasion zu hohem Verschleiß der nachfolgenden Anlagenteile und Turbinen kommt. Neben möglichen Betriebseinschränkungen hinsichtlich Volllast und Anfahren des Absenkziels sind Probleme an Laufrädern, frühzeitige Instandhaltungsarbeiten und gegebenenfalls Probleme beim Kühlwassersystem die negativen und kostenintensiven Folgen. Die erhöhte hydroabrasive Abnutzung ist auch bei Maßnahmen wie einer Sedimentweiterleitung über das Triebwasser zu 
beachten. Diesbezüglich besteht ein hohes operationales (betriebliches) Optimierungspotenzial, das auf die jeweiligen Sedimenteigenschaften abgestimmt werden sollte. Um hier verbesserte Lösungen $\mathrm{zu}$ erzielen, bedarf es der integrativen Verschneidung der vorhandenen Erfahrungen der Wasserkraftbetreiber und Anlagenhersteller mit wissenschaftlichen Monitoringdaten.

- Aus der bisherigen Erfahrung von Wasserkraftbetreibern im Alpenraum ist die regelmäßige Entlandung durch Speicherspülung bzw. -entleerung eine effiziente und zudem nachhaltige Sedimentbewirtschaftungsmaßnahme. Diese gut geeignete Entlandungsmethode hat neben zahlreichen Vorteilen jedoch den Nachteil, dass damit eine Belastung der Unterliegerstrecke durch Schwebstoffe einhergehen kann und diese in Relation zur natürlich auftretenden Schwebstoffführung $\mathrm{zu}$ betrachten ist. Ähnliche ökologisch nachteilige Belastungen der flussab gelegenen Gewässerstrecke könnten ebenfalls mit Maßnahmen wie der Zugabe von Sedimenten in das Triebwasser und dem Durchleiten von Trübeströmen auftreten. Die Prozesse dieser nachhaltigen Sedimentmanagementkonzepte müssen hinsichtlich ihrer Auswirkungen wissenschaftlich untersucht werden, um einerseits negative Folgewirkungen $\mathrm{zu}$ vermeiden und andererseits deren Effizienz zur Optimierung der Wasserkraftnutzung zu steigern.

In jedem Fall sind mit dem veränderten Feststofftransport betriebliche Einschränkungen und hohe Aufwendungen für die Wasserkraftbetreiber verbunden, die mit zunehmender Bewilligungsdauer den eigentlichen Zwecken der geplanten Nutzung abträglich sind und ein nachhaltiges Sedimentmanagement erfordern.

Um die Auswirkungen zu reduzieren oder zumindest zeitlich zu verzögern, wurden und werden bereits zahlreiche präventive und retroaktive Maßnahmen gegen die Verlandung - im Einzugsgebiet, im Stausee und an der Talsperre (Schleiss und Oehy 2002) - durchgeführt. Darunter sind jene Konzepte, die ein nachhaltiges Sedimentmanagement zum Ziel haben, zweifellos ökologisch und ökonomisch am sinnvollsten.

Auf Seiten der Betreiber wurde in der Vergangenheit der Verlandungs- problematik vielfach oft $\mathrm{zu}$ wenig Beachtung beigemessen, sodass infolge mangelnder Konsequenz in der Umsetzung, geänderter Rahmenbedingungen wie nicht mehr nutzbare Deponiestandorte sowie teilweise auch eine nicht nachhaltige Wahl der Maßnahmen der langfristige Erfolg bisher bei vielen Speicheranlagen nicht gegeben ist. Doch auch die zunehmende Sensibilisierung bei ökologischen Themen, die schwierigen rechtlichen Genehmigungsverfahren - insbesondere für Dauerbewilligungen - sowie der zum Teil nicht vorhandene wissenschaftliche Hintergrund standen bisher oft einer ausreichenden Umsetzung nachhaltiger Entlandungsmaßnahmen entgegen.

Die aktuellen Forschungstätigkeiten im Rahmen des neuen CD-Labors Sediment zu den Fragestellungen eines besseren Prozessverständnisses, Erkenntnissen in den Wechselwirkungen mit der (Gewässer-)Ökologie, innovativer Maßnahmenfindungen und verbesserten Monitoringmethoden sind diesbezüglich sehr wichtig. Dabei wird zusätzlich zur Grundlagenforschung in enger Zusammenarbeit mit betroffenen Wasserkraftbetreibern anhand von Fallbeispielen eine Optimierung von Maßnahmen angestrebt.

Nachfolgend sind exemplarisch und ohne Anspruch auf Vollständigkeit drei Beispiele für aktuelle Entlandungsmethoden bei österreichischen Speichern im alpinen Raum aufgeführt.

\subsection{Speicher Raggal}

Der Speicher Raggal dient als Oberbecken der Kraftwerksgruppe an der Lutz der Vorarlberger Kraftwerke AG; im November 1967 wurde der Kraftwerksbetrieb aufgenommen. Durch die Errichtung einer rund $48 \mathrm{~m}$ hohen Gewichtsmauer wurde ein etwa $2,3 \mathrm{~km}$ langer Wochenspeicher mit einem Gesamtinhalt von ca. 2,4 Mio. $\mathrm{m}^{3}$ und einem Nutzinhalt von 2,0 Mio. $\mathrm{m}^{3}$ geschaffen. Der nicht bewirtschaftbare Totraum des Speichers wurde bereits in den ersten 20 Jahren durch die Ablagerung von Feinsedimenten und Geschiebe bis auf Höhe des Triebwassereinlaufs aufgefüllt. Mittlerweile beeinträchtigen $620.000 \mathrm{~m}^{3}$ Anlandungen an Sedimenten auch den bewirtschaftbaren Stauraum. Mit der im Jahr 1986 in Betrieb genommenen Saugbaggeranlage konnten die vorhandenen Verlandungen zum Teil entfernt werden; dennoch betrug die aktuelle Zunahme an Feststoffeintrag zuletzt etwa $16.000 \mathrm{~m}^{3} / \mathrm{Jahr}$. Zusätzlich konnte aufgrund der Gefahr der Verlegung des Staumauergrundablasses, der neben dem leistungsfähigeren Hauptgrundablass als zweites Entleerorgan zur Verfügung steht, bei den behördlich vorgeschrieben Entleerungen 2005 und 2008 die für eine Kollaudierung erforderliche Kontrolle nicht zufriedenstellend durchgeführt werden.

Deshalb wird seit Mai 2018 ein befristetes Feststoffbewirtschaftungskonzept mit einem neuen Schwimmbagger (Abb. 1) umgesetzt, welcher die dicht gelagerten Feststoffe mit einem Zweischalengreifer mechanisch löst und dann mittels einer Förderleitung und Zusatzdotierung ins Unterwasser zugibt. Innerhalb von jährlich sechs Wochen wird nun mit der im Vergleich zur alten Saugbaggeranlage doppelten Förderleistung gebaggert. Die Halbierung der Baggerzeiten gegenüber dem ursprünglichen Konzept stellt eine deutliche ökologische Verbesserung dar. Zusätzlich wurde befristet bis Ende 2022 bewilligt, hochwasserbedingte Trübeströme durch den Speicher mit Abgabe über den Staumauergrundablass $\mathrm{zu}$ leiten. Die Maßnahme wird auch durch eine umfassende Beweissicherung begleitet.

Im ersten Betriebsjahr konnte mit der neuen Schwimmgreiferanlage trotz Anfangsschwierigkeiten bereits eine größere Menge an Feststoffen entnommen werden als in den Jahren zuvor. Die Feststoffbelastungen in der Lutz blieben während der Baggerarbeiten und auch der Nachspülungen unter dem bewilligten Richtwert. Mit weiteren Verbesserungen der Betriebsstabilität und Effizienz der Baggeranlage besteht Zuversicht, die Verlandung des Speichers Raggal langfristig in Grenzen zu halten sowie die Funktionstüchtigkeit der Grundablässe durch Freihaltung von Geschiebe dauerhaft sicherzustellen.

\subsection{Speicherbecken Rodund}

Die drei Pumpspeicherbecken Rodund mit einem Speichervolumen von insgesamt 2,1 Mio. $\mathrm{m}^{3}$ sind Unterwasserbecken der Kraftwerke Rodund I und II der Vorarlberger Illwerke AG. Der Nutzinhalt war vor der Umsetzung des neuen Entlandungskonzepts durch rund $350.000 \mathrm{~m}^{3}$ Sedimentablagerungen verkleinert. Jährlich kamen bis zu $20.000 \mathrm{~m}^{3}$ Sedimente aus der Fassung 


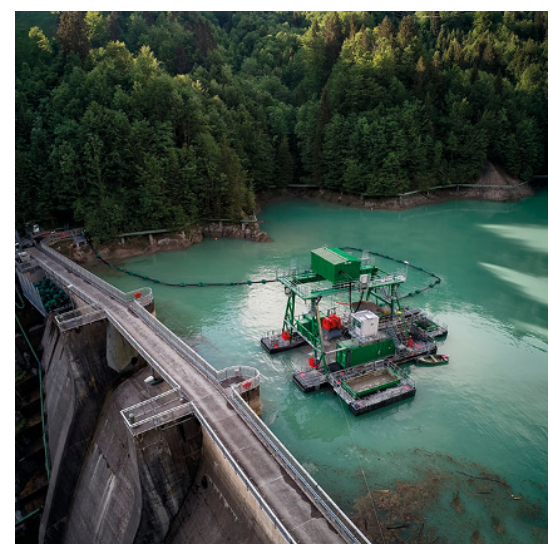

Abb. 1 Speicher Raggal, Juli 2018, neuer Schwimmbagger im Betrieb (Foto: Fa. Idreco)

an der Ill hinzu. Eine mechanische Entfernung der Sedimente wurde aus energiewirtschaftlichen Gründen, mangels geeigneter Deponien und entsprechend hoher Entsorgungskosten verworfen.

Im Zuge des Alternativkonzepts, das sich am natürlichen Sedimenttransport der Ill orientiert, wurde von 2012 bis 2017 eine Lösung mittels Saugbagger und einer Beigabe der Schlufffraktionen in das Triebwasser des Walgauwerkes als Unterlieger mit einer Gesamtentlandung von rd. $250.000 \mathrm{~m}^{3}$ umgesetzt. Die Sediment-Rückgabe erfolgte erst bei gemessener Konzentration in der Ill von $50 \mathrm{mg} / \mathrm{l}$. Die Abgabekonzentration orientierte sich an den tatsächlich gemessenen Trübewerten und durfte abgesehen von Hochwasserereignissen maximal $800 \mathrm{mg} / \mathrm{l}$ betragen. Dies ergab eine starke Abhängigkeit von den tatsächlichen hydrologischen Bedingungen wie Winterniederschlag und Schneeschmelzeverlauf und so schwankte die jährliche Entlandungsrate stark zwischen 8000 und $100.000 \mathrm{~m}^{3}$ pro Jahr. Als weitere Erfahrung zeigte sich, dass die Abrasionsrate an den Turbinen stark von den Sedimenteigenschaften abhängt. Für eine lange Beaufschlagung mit hoher Sedimentkonzentration wurde deshalb die Turbinengeometrie angepasst und eine spezielle Schutzschicht aufgebracht. Die rund $10 \%$ Sand-Fraktionen wurden ebenfalls gelöst, über eine Zyklonanlage geschickt und wiederverwertet.

Um in Zukunft die hohen Räumungskosten bzw. den jährlichen Neueintrag zu reduzieren, wird die Illfassung seit Juni 2015 in Abhängigkeit

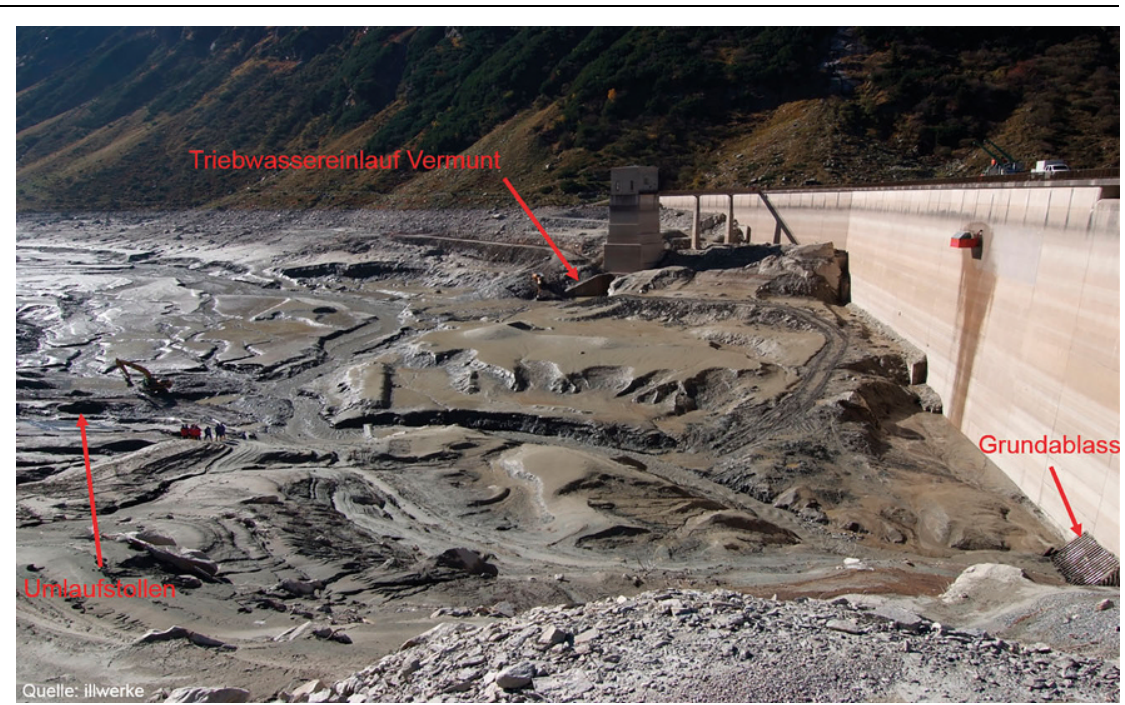

Abb. 2 Entleerter Speicher Vermunt, Okt. 2010, Sohle im Bereich vor der Sperre

von der Trübung und nach energiewirtschaftlicher Bewertung ausgeleitet.

\subsection{Speicher Vermunt}

Der Vermuntsee mit einem Nutzvolumen von rund 5 Mio. $\mathrm{m}^{3}$ dient dem Vermuntwerk seit dem ersten Vollstau 1931 als Wochenspeicher. Gleichzeitig ist der Speicher Vermunt das Unterbecken des Obervermuntwerks I und seit 2018 des deutlich größeren Pumpspeicherwerks Obervermuntwerk II und damit ein wesentlicher Bestandteil der Werksgruppe Obere Ill-Lünersee der Vorarlberger Illwerke AG. Der Vermuntsee nimmt das Wasser aus natürlichen Zuflüssen, vor allem aus dem Kromerbach und der Ill unterhalb des Silvrettaspeichers sowie weiteren Bachüberleitungen auf.

Im nordwestlichen Bereich des Speichers Vermunt befindet sich in der Nähe der Staumauer das Entnahmebauwerk für das Triebwasser des Vermuntwerks. Am Südende erfolgt die Wasserrückgabe des Obervermuntwerks I, im nordöstlichen Bereich befindet sich das Auslaufbauwerk des Obervermuntwerks II sowie die Entlastungsanlagen Grundablass und Umlaufstollen mit entsprechenden Rechen und Absperrorganen (Abb. 2).

Die Verlandung im Speicher Vermunt beträgt seit Bestehen rund 0,44 Mio. $\mathrm{m}^{3}$ mit einem mittleren jährlichen Neueintrag von rd. $6000 \mathrm{~m}^{3}$. Gröbere Fraktionen landen vor allem im Bereich der Stauwurzel an; Schluff und Feinsand setzt sich im gesamten Speicherbereich ab. Im Zuge der Tiefabsenkungen beim Bau des Obervermunt- werks II wurde Material in der Größenordnung von etwa $50.000 \mathrm{~m}^{3}$ von der Stauwurzel Richtung Staumauer insbesondere vor die Entleerorgane verlagert. Mit aufwendigen Sedimentumlagerungen innerhalb des Speichers Vermunt und regelmäßigen Funktionstests des Grundablasses sowie des Umlaufstollens konnten die Entlastungsorgane freigeräumt und deren Funktionalität weiterhin gewährleistet werden.

Um dies künftig auch nachhaltig sicher stellen zu können, wird ein neues Feststoffbewirtschaftungskonzept vorbereitet, das in regelmäßigen Intervallen und $\mathrm{zu}$ den behördlich vorgeschriebenen Speicherentleerungen die nächste geplante findet 2024 statt - einen Durchleitbetrieb und freien Durchfluss über den Grundablass mit kontrolliertem Sedimentaustrag vorsieht. Unter Einhaltung festgelegter Randbedingungen, entsprechend vorbereiteter Maßnahmen und begleitendem Monitoring soll mit dieser Methode eine dauerhafte Begrenzung der Sedimentverlandung erreicht werden. Eine entscheidende Fragestellung kommt dabei den Auswirkungen der durch die Betriebsweise des Obervermuntwerks II geänderten Stauraumbewirtschaftung und der daraus resultierenden Verlandungssituation zu. Hier werden erst die weiteren Betriebsjahre entsprechenden Aufschluss bringen können. 


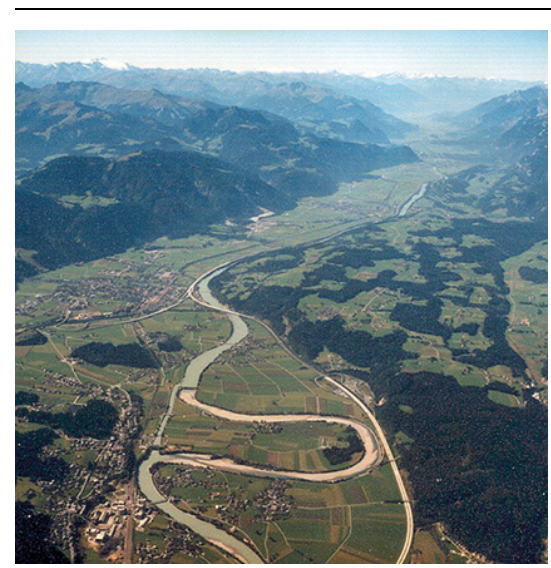

Abb. 3 Innkraftwerk Kirchbichl

\section{Flusskraftwerke}

\subsection{Feststoffmanagement am Tiroler Inn}

Der Tiroler Inn weist von der Staatsgrenze zur Schweiz bis zur Grenze nach Deutschland eine Länge von ca. $200 \mathrm{~km}$ auf. Das Einzugsgebiet des Inns nimmt entlang dieser Fließstrecke vor allem durch die Sanne, die Pitze, die Ötztaler Ache, die Sill, den Ziller und die Brixentaler Ache von ca. $2000 \mathrm{~km}^{2}$ auf ca. $9500 \mathrm{~km}^{2}$ stark zu. Umgekehrt verhält es sich mit dem Längsgefälle, das von rund $5 \%$ im Oberlauf auf rund $1 \% 0$ im Unterlauf abnimmt. Entlang der Fließstrecke des Tiroler Inns betreibt die TIWAG drei Flussstauhaltungen, deren Feststoffmanagement im Folgenden beschrieben wird:

- Die zur Kraftwerksanlage Prutz-Imst gehörige Wehranlage Runserau im Oberlauf staut den Inn um maximal $10 \mathrm{~m}$ auf und erzeugt so ein Stauraumvolumen von ca. $850.000 \mathrm{~m}^{3}$. Der ca. $3 \mathrm{~km}$ lange Stauraum der Wehranlage Runserau wird im Tagesschwellbetrieb bewirtschaftet, wobei sich die Wasserspiegelhöhe um maximal 5,5m verändern kann. Das aus dem Stauraum entnommene Triebwasser wird über einen ca. $12 \mathrm{~km}$ langen Stollen dem Krafthaus in Imst zugeführt, wodurch unterhalb der Wehranlage Runserau eine ca. $25 \mathrm{~km}$ lange Restwasserstrecke vorhanden ist. Vor allem in den abflussreichen Sommermonaten kommt es zu einem beträchtlichen Feststoffeintrag in den Speicher Runserau. Während sich das grobe Geschiebe (durchschnittlich ca. $25.000 \mathrm{~m}^{3}$ pro Jahr) aufgrund der verminderten Fließgeschwindigkeit bereits im Stauwurzelbereich ablagert, kommt es bei den feinen Schwebstoffen (500.000 t/a) einerseits $\mathrm{zu}$ einem selektiven $\mathrm{Ab}$ setzprozess über den gesamten Stauraum und andererseits auch gleichzeitig zu einem Weitertransport über das Trieb- und Überwasser. Insgesamt ergeben sich im Stauraum Runserau beträchtliche Feststoffablagerungen, welche durch regelmäßige Stauraumspülungen (üblicherweise jährlich) wieder aus dem Stauraum ausgetragen werden und damit im weiteren Flusssystem unverändert zur Verfügung stehen. Diese Stauraumspülungen werden im Zuge von Hochwasserereignissen durch vollständige Öffnung der Wehranlage und Herstellung des freien Abflusses vorgenommen. Da die Hochwasserereignisse auch natürlicherweise mit hohen Schwebstoffkonzentrationen einhergehen, werden die im Zuge der Stauraumspülungen aus dem Stauraum ausgetragenen Schwebstoffe somit in den dafür typischen Zeiträumen weitertransportiert. Aufgrund der Gefälleverhältnisse entsteht bei freiem Abfluss eine beträchtliche Transportkapazität, sodass selbst das gröbere Geschiebe leicht mobilisiert und ins Unterwasser weitertransportiert werden kann. Die Gesamtdauer einer Stauraumspülung samt Ab- und Aufstauphase beträgt ca. ein bis drei Tage. Im langjährigen Mittel werden Stauraumspülungen etwa 0,8 mal pro Jahr durchgeführt. Die durch den Feststoffeintrag bzw. die Stauraumspülungen hervorgerufenen Sohländerungen werden mittels Echolotmessungen (Profilmessungen im Abstand von 100 bis $200 \mathrm{~m}$ ) ermittelt und hinsichtlich Speichervolumenänderung ausgewertet.

- Die beiden im Unterlauf des Tiroler Inn gelegenen Flussstauhaltungen der Kraftwerke Kirchbichl und Langkampfen liegen unmittelbar hintereinander und bilden den Anfang einer durchgehenden Kraftwerkskette entlang des Inn bis zur Donau. Während das Kraftwerk Kirchbichl als Ausleitungslaufkraftwerk mit Triebwasserkanal zur Abkürzung der ca. $3,5 \mathrm{~km}$ langen Innschleife Kirchbichl angeordnet ist (s. Abb. 3), handelt es sich beim Kraftwerk Langkampfen um ein im Flussschlauch angeordnetes Buchtenkraftwerk. Beide Anlagen werden als Laufkraftwerke mit konstantem Oberwasserspiegel betrieben, wobei der Rückstau des Kraftwerks Langkampfen mit mehr als $7 \mathrm{~km}$ Länge bis zum Krafthaus Kirchbichl zurückreicht und der Rückstau der Wehranlagen Kirchbichl eine Länge von ca. $3 \mathrm{~km}$ erreicht. Die Stauraumvolumina betragen ca. $900.000 \mathrm{~m}^{3}$ bei Kirchbichl und ca. 3,5 Mio. $\mathrm{m}^{3}$ bei Langkampfen. Um die ca. $3,3 \mathrm{~km}$ lange Unterwassereintiefungsstrecke des Kraftwerks Langkampfen von Sohlanlandungen frei $\mathrm{zu}$ halten und gleichzeitig die anschließende VERBUND-Kraftwerkskette vor Geschiebeeinträgen zu schützen, gibt es im Rückstaubereich ca. $500 \mathrm{~m}$ flussauf des Kraftwerks eine Kiesfalle in Form einer ca. $400 \mathrm{~m}$ langen und ca. $6 \mathrm{~m}$ tiefen Sohleintiefung. Ähnlich wie bei der vorher beschriebenen Stauhaltung am Wehr Runserau kommt es auch bei der Stauhaltung Kirchbichl vor allem in den abflussreichen Sommermonaten zu einem beträchtlichen Eintrag von Geschiebe und Schwebstoffen. Dabei stellen vor allem die durchschnittlich rund $140.000 \mathrm{~m}^{3}$ Geschiebe pro Jahr eine große Herausforderung für das Sedimentmanagement dar. Damit der Geschiebetransport bestmöglich beherrscht werden kann, ist die Betriebsweise der Kraftwerke Kirchbichl und Langkampfen im Hochwasserfall aufeinander abgestimmt. Diese Betriebsweise sieht vor, dass bei erforderlicher Stauraumspülung im Kraftwerk Kirchbichl (üblicherweise ab Abflüssen von $700-800 \mathrm{~m}^{3} / \mathrm{s}$ ) auch der Stau in Langkampfen gelegt und der Stauraum bei freien Abfluss für eine Dauer von fünf bis zehn Tagen gespült wird. Dabei wird bei ansteigender Hochwasserwelle zuerst in Langkampfen, dann erst in Kirchbichl der freie Abfluss hergestellt und am Spülende bei abklingender Hochwasserwelle in umgekehrter Reihenfolge zuerst am Kraftwerk Kirchbichl und dann erst in Langkampfen der Aufstau vorgenommen. Damit wird sichergestellt, dass Geschiebe, welches in den Stauraum Langkampfen gelangt möglichst direkt bis in die Kiesfalle vor dem Kraftwerk weitertransportiert wird. Im langjährigen Mittel werden etwa 1,5 mal pro Jahr Stauraumspülungen durchgeführt, in einzelnen Jahren bis zu dreimal. Neben den abgestimmten Stauraumspülungen sieht das Sedimentmanagement zusätzlich auch jährliche 


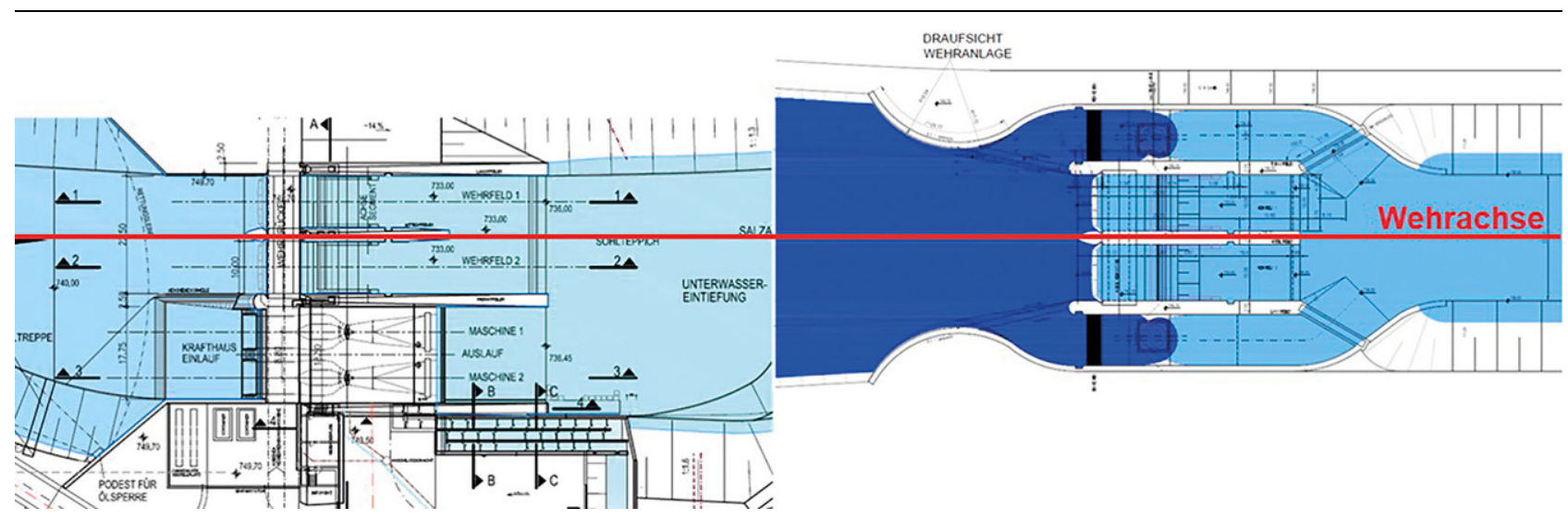

Abb. 4 Vergleichzwischen der buchtenartigen Bauweise (links) und den seitlich angeordneten Turbinensätzen (rechts). Zur Verdeutlichung ist die Wehrachse markiert. Deutlich wird die geringe Aufweitung im Unterwasser im rechten Bildteil (aus: Badura 2018)

Geschiebeentnahmen aus der Innschleife Kirchbichl und aus der Kiesfalle vor. Während die Geschiebeentnahme aus der Innschleife Kirchbichl mit herkömmlichen Erdbaugeräten vorgenommen werden kann, kommt bei der Räumung der Kiesfalle eine Entnahme unter Wasser mittels Saugbaggerung zum Einsatz. Dabei sind nicht nur Saugtiefen von bis zu $15 \mathrm{~m} \mathrm{zu}$ bewältigen, sondern auch starke Verschleißerscheinungen bei den Fördereinrichtungen der Saugbaggeranlage durch das abrasive Geschiebe. Da die beiden Staustufen Kirchbichl und Langkampfen am Beginn einer langen Kraftwerkskette liegen, müssen die zur Sedimentweitergabe im Hochwasserfall notwendigen Abflussänderungen in der $\mathrm{Ab}$ und Aufstauphase sehr vorsichtig und moderat durchgeführt werden. Um dies sicherstellen $\mathrm{zu}$ können, ist eine entsprechend lange Vorlaufund Reaktionszeit erforderlich, wofür ein vollautomatisches Hochwasserprognosemodell als Hilfestellung aufgebaut wurde.

\subsection{Verbesserung der Geschiebedurchgängigkeit durch innovative Anlagenkonzeption}

Bereits die grundlegende Konzeption einer Wasserkraftanlage hat maßgebliche Auswirkungen auf die Feststoffdurchgängigkeit. Beim 2019 fertiggestellten Salzachkraftwerk Gries von VERBUND und Salzburg AG wurde besonders auf die Geschiebedurchgängigkeit des Kraftwerks geachtet. Bei stark geschiebeführenden, alpinen Flüssen wie der Salzach kommt es bei der bisher üblichen Bauweise eines Buchtenkraft- werks aufgrund der Querschnittsaufweitung zu Anlandungen im Unterwasser in unterschiedlichster Ausprägung. Die gewählte, seitliche Anordnung der Maschinensätze beim Kraftwerk Gries wurde im hydraulischen Modellversuch untersucht und kommt ohne nennenswerte Aufweitungen im unmittelbaren Anlagebereich aus (Abb. 4), sodass Anlandungen durch dieses Kraftwerkskonzept vermieden werden.

Zur Bestimmung des Geschiebetransports kommt das innovative Messsystem SIS (Sediment Impact Sensor), das an der TU Graz, Institut für Wasserbau und Wasserwirtschaft entwickelt wurde, zum Einsatz (Sass et al. 2015). Die damit gewonnenen Daten stehen dann zur wissenschaftlichen Analyse zur Verfügung und können somit auch zur Verbesserung des Prozessverständnisses beitragen

\subsection{Stauraumbewirtschaftung und}

Hydrologie an der Mittleren Salzach

Wie zahlreiche Beobachtungen und die angeführten Fallbeispiele zeigen, verursachen Laufkraftwerke in der Regel eine Unterbrechung des Feststoffkontinuums. Dies insbesondere dann, wenn es sich um entsprechend lange Stauhaltung oder Kraftwerksketten handelt. Koordinierte Stauraumspülungen bei Hochwasserereignissen stellen hier, wie auch die Erfahrungen an der Kraftwerkskette Mittlere Salzach zeigen, eine effiziente Methode zur Aufrechterhaltung eines weitgehend stabilen Feststoffhaushalts dar.

Die Kraftwerkskette Mittlere Salzach besteht aus sieben Laufkraftwerken, welche unmittelbar aneinander anschließen und im Hochwasserfall ent- sprechend einer gemeinsamen Wehrbetriebsordnung betrieben bzw. zur Herstellung des freien Durchflusses koordiniert abgestaut werden. Im Durchschnitt erfolgt so jährlich eine Stauraumspülung, wodurch, wie die jährlichen Vermessungen der Flusssohle zeigen, weitgehend stabile Anlandungsverhältnisse zur Gewährleistung der Hochwassersicherheit ermöglicht werden. Stauraumspülungen dauern in der Regel zwischen drei und fünf Tagen, wobei sich der Durchgang der eigentlichen Hochwasserwelle oft auf relativ kurze Zeiträume von unter $24 \mathrm{~h}$ beschränkt und die verbleibende Zeit der Nachspülung dient. Zunehmend schwierig gestalten sich die Spülungen angesichts der Tatsache, dass mittlerweile Hochwasserereignisse im Einzugsgebiet der Mittleren Salzach häufig aus relativ kurzfristig auftretenden flächig begrenzten Niederschlägen resultieren, welche dadurch von steil ansteigenden Hochwasserwellen mit kurzer Dauer charakterisiert sind.

Entsprechende Auswertungen der Abflussdaten des Salzachpegels in Golling für den Zeitraum 1977 bis 2017, basierend auf 15-Minuten-Mittelwerten, zeigen, dass die Anzahl der jährlichen Hochwasserereignisse $>450 \mathrm{~m}^{3} / \mathrm{s}$ (entspricht HQ1) leicht rückläufig (Abb. 5) ist, jedoch die jährlich kumulierte Wellendauer dieser Ereignisse um etwa $40 \%$ abgenommen hat (Abb. 6).

Angesichts dieser Entwicklung ist für eine effiziente Stauraumentlandung ein zuverlässiges Hochwasserprognosemodell, wie dieses auch für die Mittlere Salzach existiert, und eine entsprechende Wehrbetriebsordnung von großer Bedeutung. Mit der 2008 für die Anlagen der Mittleren Salzach be- 


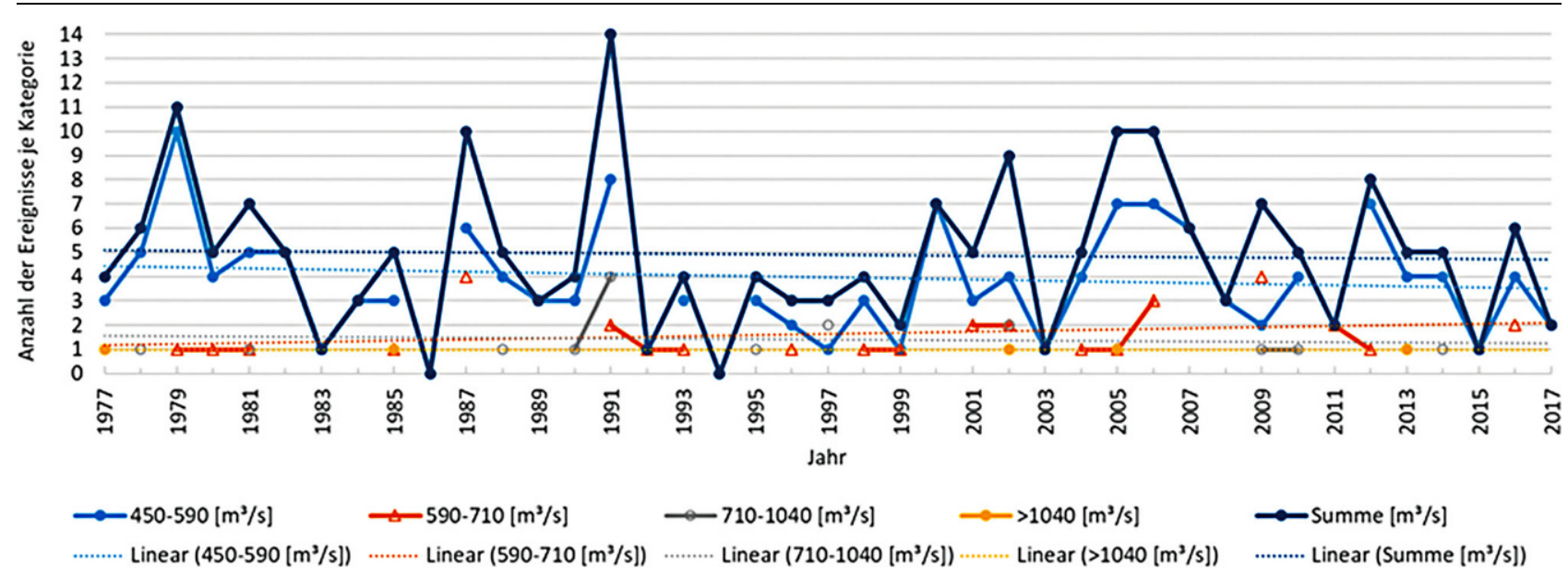

Abb. 5 Salzachpegel Golling: jährliche Anzahl der Hochwässer in Kategorien als Absolutwerte und linearer Trend

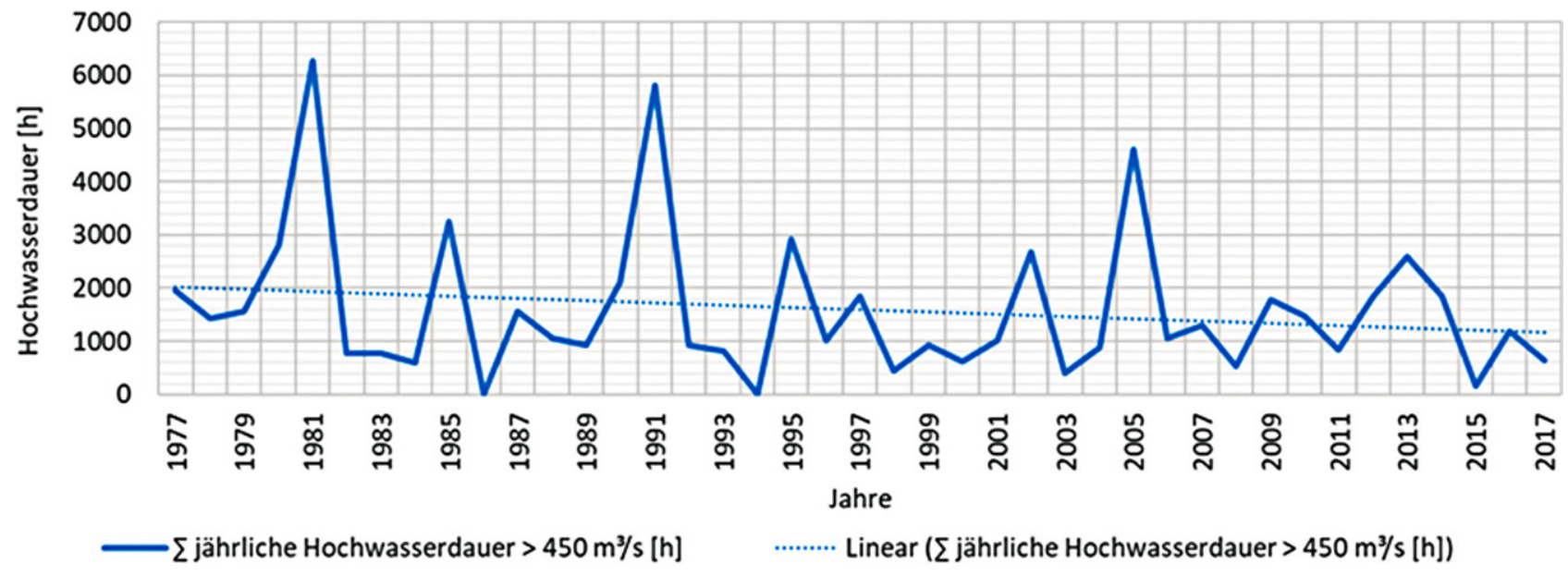

Abb. 6 Salzachpegel Golling: Kumulierte jährliche Hochwasserdauer $>450 \mathrm{~m}^{3} / \mathrm{s}$ in Stunden

willigten Wehrbetriebsordnung konnte die erforderliche Absenkdauer bis zum Erreichen des freien Durchflusses an der gesamten Kraftwerkskette von gesamt etwa 32 auf $13 \mathrm{~h}$ reduziert werden. Entsprechend der Abflussprognosen sieht die Wehrbetriebsordnung auch die Möglichkeit einer Vorabsenkung der Stauräume um bis zu zwei Meter vor, wodurch sich im Anlassfall die Dauer bis zum Erreichen des freien Durchflusses nochmals deutlich reduziert.

\subsection{Kleinwasserkraftwerk Hausmening an der Ybbs}

Mit Bescheid vom 9. Mai 2012 erteilte das Amt der NÖ Landesregierung WIEN ENERGIE die wasserrechtliche Bewilligung zur Errichtung des Kraftwerkes Hausmening an der Ybbs nahe Amstetten. Der neue Standort liegt rund $350 \mathrm{~m}$ flussab des früheren Kraftwerks The- resienthal und ersetzt unter Nutzung beider Gefällestufen die alten Kraftwerke Theresienthal und Hofmühle. Beim Kraftwerk Hausmening (Abb. 7) wurde auf einer Länge von ca. $940 \mathrm{~m}$ bis zum alten Standort Hofmühle eine Unterwassereintiefung durchgeführt und eine Niederwasserrinne errichtet.

Die Aushubarbeiten zur Unterwassereintiefung begannen im September 2012 zeitgleich vom oberen und unteren Ende. Aushubmaterial im Ausmaß von ca. $125.000 \mathrm{~m}^{3}$ wurde verführt. Mit Anfang März 2014 waren die Maßnahmen zur Herstellung der Unterwasserstrecke weitestgehend abgeschlossen. Im Rahmen einer baugeologischen Aufnahme der Flusssohle wurden die Höhenlage des Schliers sowie die Mächtigkeit der überlagernden Flussschotter dokumentiert. Bei der Aufnahme der Flussquerprofile am 5. März 2014 zeigte sich eine weitgehende Übereinstim- mung der Sollprofile mit dem Istbestand.

Nach weitgehendem Abschluss der Bauherstellung erfolgte die Nass-Inbetriebnahme des Kraftwerks Hausmening im April/Mai 2014. Am 16. Mai 2014 ereignete sich ein knapp 30-jährliches Hochwasser mit einer Abflussspitze von rund $850 \mathrm{~m}^{3} / \mathrm{s}$. Wie sich an mehreren Standorten zwischen Waidhofen an der Ybbs und Amstetten zeigte, war das Hochwasserereignis generell von einer hohen Substrat- und Sohldynamik am Mittel- und Unterlauf der Ybbs geprägt. Im Bereich des Kraftwerkes Hausmening wurde die Geschiebeführung durch eigendynamische morphologische Entwicklungen bei einem flussauf im Rahmen des LIFE+Projektes Mostviertel Wachau neu geschaffenen Nebenarm verstärkt, welcher sich im Zuge des Hochwasserereignisses durch Erosion deutlich verbreiterte. Generell 


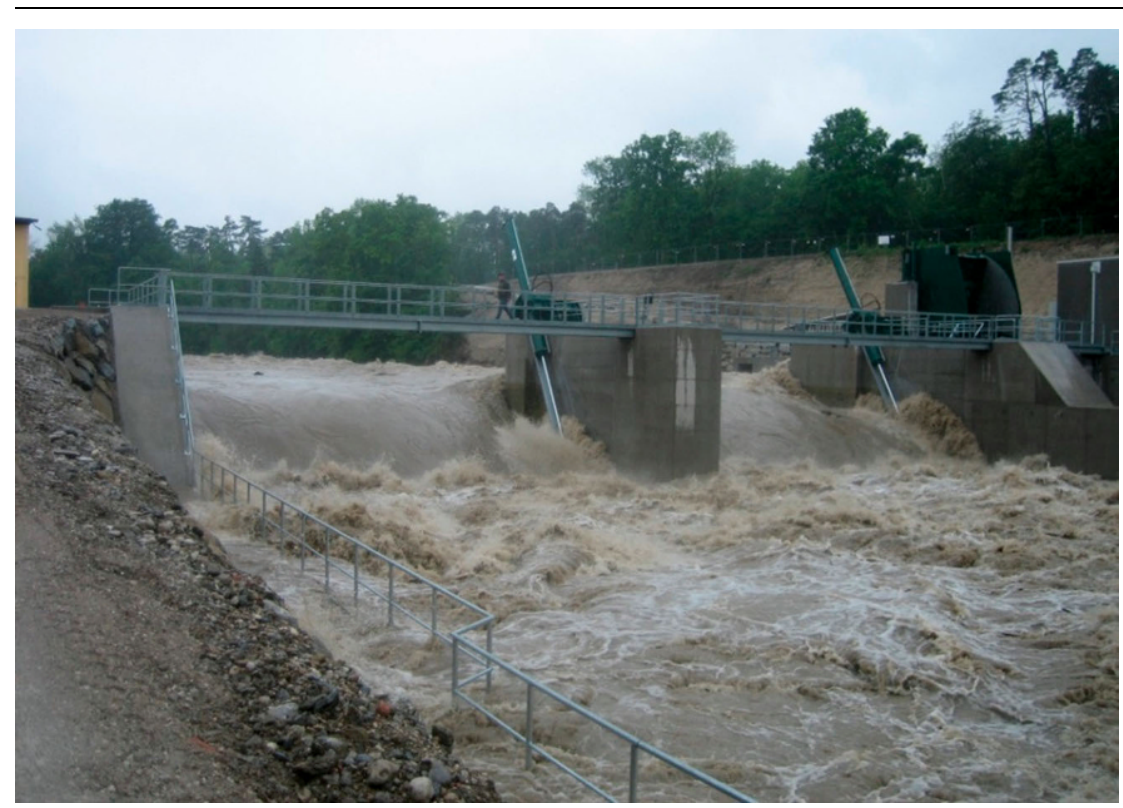

Abb. 7 Hochwasserabfluss am Kraftwerk Hausmening an der Ybbs vom 16. Mai 2014

zeigte sich nach dem Hochwasser in der Unterwasserstrecke eine flächige Anlandung von Schottersubstrat, speziell auch unterhalb des Turbinenauslaufs.

Mitte August 2014 wurde im Bereich unmittelbar unterhalb des Kraftwerks die Tiefenrinne des Turbinenauslaufs wiederhergestellt. Das Material wurde dabei rechtsufrig umgelagert. Am 10. November 2014 erfolgte eine Aufnahme der Querprofile mit zusätzlichen Hilfsprofilen. Die Gegenüberstellung der Ergebnisse mit den Aufnahmen vom 5. März 2014 zeigt in der gesamten Unterwasserstrecke starke Anlandungen infolge des Hochwassers vom Mai 2014. Der Vergleich mit dem projektierten Plansoll zeigt für die einzelnen Querprofile Anlandungen von im Mittel 0,3 bis $0,9 \mathrm{~m}$. Die Erzeugungsverluste infolge der um rund $10 \%$ reduzierten Fallhöhe und damit zudem nicht mehr im optimalen Bereich gelegenen Turbinenkennfelder waren erheblich.

Die Wiederherstellung der Sollprofile wurde Anfang Februar 2015 abgeschlossen. Seitdem bleiben die Profile im Unterwasser trotz kleinerer Hochwasserereignisse unterhalb von $\mathrm{HQ}_{5}$ weitestgehend stabil und die volle Fallhöhe des Kraftwerks steht für die Energieerzeugung zur Verfügung. Bei großen Hochwasserereignissen ist eine Anlandung auch künftig nicht auszuschließen, wobei hier noch Forschungsbedarf bezüglich der tatsächlichen Ausprägung in Abhängigkeit von der Hochwasserwelle besteht.

\subsection{Feinsedimentmanagement an der österreichischen Donau}

Die Kraftwerkskette an der österreichischen Donau zwischen Jochenstein an der Staatsgrenze zu Deutschland und dem Donaukraftwerk Freudenau in Wien ist nur durch die freie Fließstrecke der Wachau unterbrochen. Die österreichische Donau ist seit jeher ein stark schwebstoffführender Fluss; der Schwebstoffhaushalt wird vor allem durch das hochalpine Einzugsgebiet des Hauptzubringers Inn geprägt. Nach dem großen Donauhochwasser des Jahres 2013 wurden der Schwebstoffhaushalt der Kraftwerkskette und die Möglichkeiten eines Feinsedimentmanagements im Rahmen der Arbeitsgruppe „Task Force Donau“ im damaligen Bundesministerium für Land- und Forstwirtschaft, Umwelt und Wasserwirtschaft einer detaillierten Analyse unterzogen (BMLFUW 2015b).

Sowohl bei Normalwasserführung als auch bei kleineren Hochwässern bis zu etwa zehnjährlichen Ereignissen bewirkt der Kraftwerksstau in Summe über die gesamte Kraftwerkskette die Ablagerung von Schwebstoffen in den Stauräumen. Die Kraftwerkskette wirkt in diesen langen Zeiträumen als Schwebstoffsenke, der Schwebstoffgehalt stromab der Kette wird im Vergleich zum unbeeinflussten Zustand verringert.

Nur bei großen Hochwässern und der dann erforderlichen teilweisen
Staulegung, die dazu dient, das Hochwasser sicher durch den Stauraum zu leiten und unzulässige Anhebungen der Hochwasserspiegellagen im Rückstauraum $\mathrm{zu}$ verhindern, erfolgt eine teilweise Remobilisierung von Feinsedimenten. In diesen kurzen Zeiträumen von jeweils nur wenigen Tagen wird die Schwebstofffracht im Bereich der Kette und stromab temporär gegenüber dem Zustand vor Kraftwerkserrichtung deutlich vergrößert. Der remobilisierte Anteil liegt bei Extremhochwässern an der österreichischen Donau in der Größenordnung von $50 \%$ der Gesamtschwebstoffführung.

Zwischen den großen Hochwässern strebt die Verlandung der Stauräume einem theoretischen Endverlandungszustand zu, der aber wegen der wiederholten Remobilisierung bei Extremhochwässern bislang kaum erreicht wurde. Abb. 8 illustriert die zeitliche Entwicklung der Stauraumverlandung am Beispiel des Donaukraftwerkes Aschach, dessen über $40 \mathrm{~km}$ langer Stauraum für den Schwebstoffhaushalt der gesamten Staukette von zentraler Bedeutung ist.

Die Verlandungsentwicklung in den Donaustauräumen wird von VERBUND und viadonau durch regelmäßige Sohlgrundvermessungen dokumentiert. Darauf aufbauend prüft die zuständige Anlagenbehörde, das Bundesministerium für Nachhaltigkeit und Tourismus (BMNT), ob die projektgemäße Hochwassersicherheit weiterhin gegeben ist oder ob allenfalls Anpassungsmaßnahmen (Dammaufhöhungen, Änderung der Höhenlage der Überströmstrecken, Baggerungen, Abänderung der Wehrbetriebsordnungen) erforderlich sind. Nach der bisherigen jahrzehntelangen Erfahrung mit der Donaukraftwerkskette hat die Verlandung der Stauräume durch Feinsedimentation keine nachteiligen Auswirkungen auf die Hochwassersicherheit. Die erforderlichen baulichen Maßnahmen oder Anpassungen der Wehrbetriebsordnungen zur Sicherstellung des projektsgemäßen Freibords der Rückstaudämme bzw. der projektsgemäßen Hochwasserspiegellagen im Bereich von Überströmstrecken waren selbst nach Extremhochwässern wie 2002 und 2013 sehr gering bzw. lokal beschränkt.

In den Überflutungsräumen entlang der österreichischen Donau, insbesondere in den großen Beckenlandschaften, gelangen die Schwebstoffe auch in die Vorländer. Sobald das Donauwasser über die Ufer tritt, geht die Fließge- 


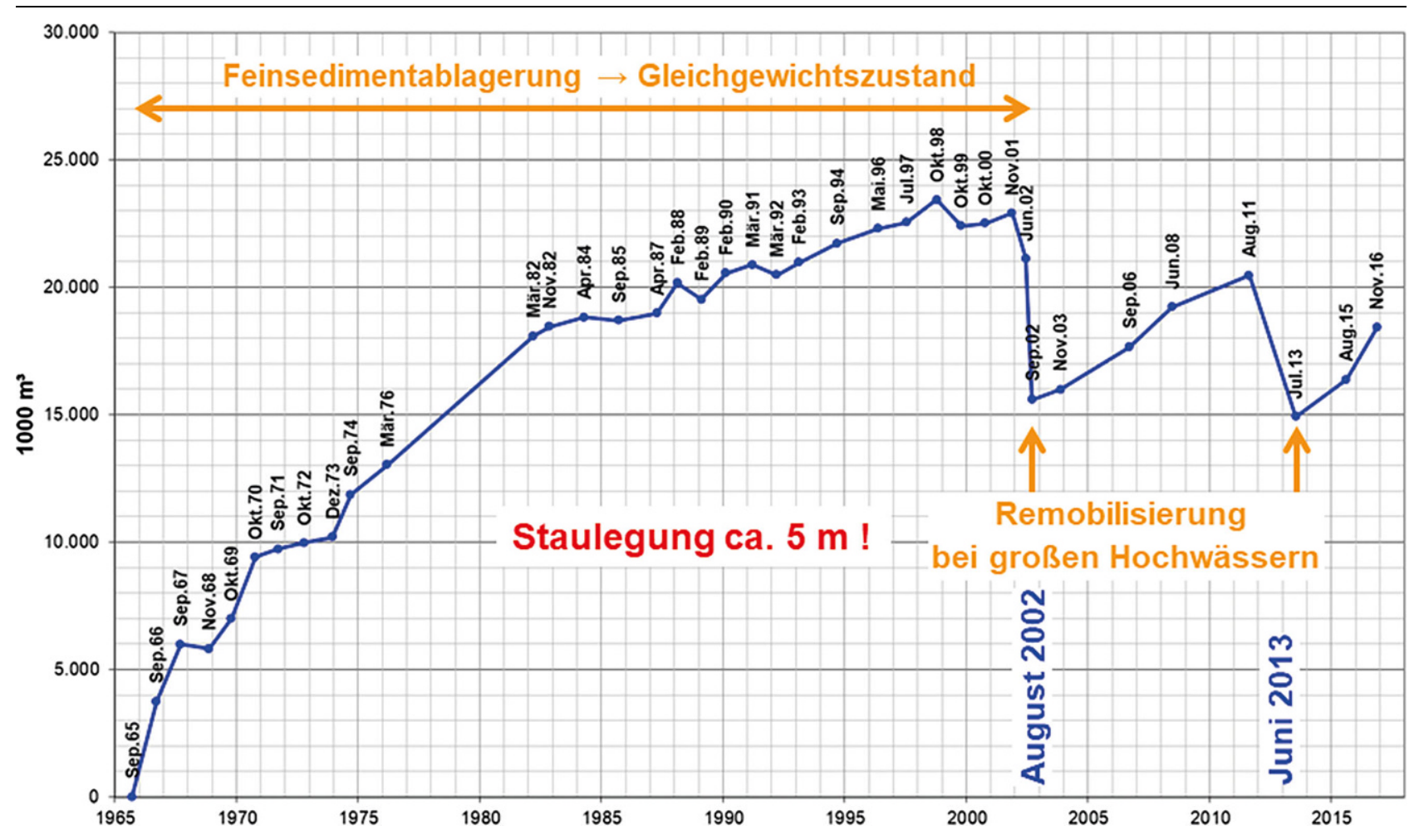

Abb. 8 Donaustauraum Aschach: Sedimentablagerungen 1960-2016

schwindigkeit und damit die Turbulenz der Strömung stark zurück, das Schwebstofftransportvermögen sinkt drastisch ab und ein großer Teil der Schwebstoffe lagert sich im Vorland, insbesondere im unmittelbaren Uferbereich (Klasz 2014), im Nahbereich von Überströmstrecken und an Abflusshindernissen wie Busch- und Baumgruppen ab. Die flächige Verlandung in den Vorländern infolge bereits abgelaufener Hochwasser und ähnlicher Ereignisse in der weiteren Zukunft führt jedoch nach vorliegenden Grobabschätzungen auch über einen Betrachtungszeitraum von einigen Jahrhunderten nur zu geringen Auswirkungen auf die Hochwasserspiegellagen.

Zur Minderung der Feinsedimentbelastung in den Vorländern wäre es dennoch grundsätzlich wünschenswert, beide Anteile - einerseits aus der Oberliegerstrecke bzw. von Zubringern eingebrachte Schwebstoffe und andererseits in den Donaustauräumen remobilisierte Feinsedimente - zu reduzieren. Seitens der Task Force wurde dazu ein Maßnahmenkatalog entwickelt. Die vollständige oder weitgehende Räumung des in den Stauräumen abgelagerten Feinsediments durch Baggerungen wurde dabei nicht als geeignete Option angesehen, da leere Stauräume be- sonders rasch verlanden und die Maßnahme daher nicht nachhaltig wäre, für die jährlich anfallende Sedimentmenge im Nahbereich keine ausreichenden Verwertungs- bzw. Deponierungsmöglichkeiten zur Verfügung stehen und ein unverhältnismäßig hoher Aufwand verursacht würde. Allfällige Spülungen im Zuge von Hochwässern wären infolge teils erheblicher und noch im Detail zu prüfender Auswirkungen auf Unterlieger, die Donauschifffahrt, den Nationalpark Donauauen, Natur und Ökologie, Landwirtschaft, Hochwasserschutz, Energieerzeugung etc. an der österreichischen Donau kaum verträglich.

Durch Feinsedimentablagerungen bedingte lokale Beeinträchtigungen in den Überflutungsgebieten können durch Managementmaßnahmen nach Abklingen der Hochwasserwelle gemildert werden:

- Im unmittelbaren Nahbereich von baulich abgegrenzten Überströmstrecken an den Rückstaudämmen, wo eine räumlich konzentrierte Ausströmung ins Vorland erfolgt, bestehen bescheidmäßige Verpflichtungen des Kraftwerksbetreibers zur Räumung lokal verstärkter Anlandungen.
- Im Eferdinger Becken und im Machland existieren sehr gute Erfahrungen mit privatrechtlichen Übereinkommen zwischen dem Kraftwerksbetreiber und den Landwirtschaftskammern von Niederösterreich und Oberösterreich für die Abgeltung landwirtschaftlicher Schäden durch vermehrte Feinsedimentbelastung bei größeren Hochwässern infolge von Bestand und Betrieb der Donaukraftwerke. Als Grundlage für die Entschädigungsbemessung dienen Befundaufnahmen nach großen Hochwässern durch gemischte Kommissionen.

Bezüglich Remobilisierung, Transport und Ablagerung von Feinsediment bei Staulegungen ist das Prozessverständnis bei Weitem noch nicht vollständig, sodass hier im Rahmen der Task Force Forschungsbedarf identifiziert wurde, der nunmehr auch in das aktuelle CDLabor Sediment Eingang gefunden hat.

\section{Schlussfolgerungen und weiterer Forschungsbedarf}

Die Nutzung der Wasserkraft bringt in vielen Fällen eine Veränderung des Feststoffhaushalts eines Flusssystems mit sich und ist aufgrund natürlicher 
physikalischer Zusammenhänge unvermeidlich. Ökologisch negative Auswirkungen können im Vorfeld durch konstruktive Maßnahmen bei der Kraftwerksplanung sowie durch angepasste Betriebsführung jedenfalls gemildert werden. Bei vielen Wasserkraftanlagen Flusskraftwerken und Speicheranlagen - verbleibt jedoch die Notwendigkeit geeigneter und idealerweise nachhaltiger Feststoffmanagementmaßnahmen wie Stauraum- bzw. Speicherspülungen oder Kompensationsbaggerungen, um die kontinuierliche Verlandung bzw. die Feststoffdefizite im Unterlauf von Stauhaltungen und negative ökologische Konsequenzen entweder zu verhindern oder zumindest $\mathrm{zu}$ reduzieren bzw. $\mathrm{zu}$ verlangsamen. Anhand von exemplarischen Fallbeispielen bei österreichischen Wasserkraftanlagen wurden aktuelle Anwendungen von Methoden des Feststoffmanagements beschrieben, innovative Konzepte vorgestellt, vorhandene Defizite aufgezeigt und die notwendigen Verbesserungen erläutert. Dies verdeutlicht die zahlreichen Anforderungen an die Forschung inklusive eines notwendigen Erfahrungsaustauschs, um die Wasserkraftnutzung einhergehend mit der Einhaltung ökologischer Rahmenbedingungen zu optimieren.

Die wesentlichen Managementmaßnahmen bei Flussstauen sind Stauraumspülungen und Kompensationsbaggerungen - diese ggf. in Kombination mit Geschiebezugaben flussab der Kraftwerkskette. Abhängig von der Anlagengröße ist für die meisten der Zubringer zur Donau und Drau einer Stauabsenkung oder Stauraumspülung der Vorzug zu geben. Offene Fragen betreffen dabei optimierte Vorgehensweisen z.B. für Spülungen in Stauräumen oder Stauketten, um betrieblich und gewässerökologisch einen kosteneffizienten und zuverlässigen Weitertransport von Schwebstoffen und Geschiebe sicherzustellen.
Bei alpinen Speichern wurde das Sedimentmanagement vielfach aufgrund der vorhandenen Dimensionen, Toträume und Deponiemöglichkeiten anfangs nicht mit hoher Priorität gesehen. Geänderte Rahmenbedingungen durch Klimawandel, Rückgang der Gletscher und des Permafrosts und der damit verbundenen vermehrten Freigabe von Sedimenten im Kontext mit sicherheitstechnischen Überlegungen zur Freihaltung der Betriebseinrichtungen erfordern nunmehr nachhaltige Feststoffmanagementmaßnahmen. Diese sind erfahrungsgemäß im wesentlichen Spülungen und Speicherentleerungen mit entsprechendem Sedimentaustrag, die schon alleine durch sicherheitstechnische Inspektionen und zum Freihalten der Sicherheitsorgane regelmäßig durchzuführen sind. Um im Hinblick auf Gewässerökologie, Morphologie, Limnologie und Fischereibiologie optimiert vorzugehen, müssen Abläufe verbessert und einheitliche technische Standards für Spülungen mit begleitendem Monitoring entwickelt werden. Gewässerökologische Belastungsgrenzen müssen in Abhängigkeit von zeitlichen Einwirkungen und Maßnahmen wie Zusatzdotation und Nachspülungen angepasst werden.

Weitere nachhaltige Methoden bei Speichern sind die Sedimentweiterleitung über das Triebwasser, gegebenenfalls das Durchleiten von Trübeströmen und in Sonderfällen die Geschiebedurchleitung in Bypassstollen, da damit größere Anlandungen erst gar nicht zugelassen werden.

Generell ist Feststoffmanagement bei Wasserkraftanlagen heute unumgänglich. Neuanlagen müssen bereits in der Konzeption ein nachhaltiges Feststoffbewirtschaftungskonzept beinhalten. Bei Bestandsanlagen ist in einer Einzelfallbetrachtung eine nachhaltige Lösung mit der geringstmöglichen Beeinflussung der Betriebsweise anzustreben.
Dabei sollte die gesamthafte Betrachtung von Feststoffmanagementkonzepten immer flussgebietsbezogen und sektorenübergreifend sein und sämtliche Stakeholder einbeziehen.

In Hinblick auf Prozessverständnis und Maßnahmenkonzeption besteht weiterhin hoher Bedarf an Forschung und Erfahrungsaustausch, der für die österreichischen Wasserkraftbetreiber wesentlicher Anlass für die Unterstützung des von der Christian Doppler Forschungsgesellschaft 2017 eingerichteten Labors für Sedimentforschung und -management ist.

Danksagung Wir bedanken uns für die finanzielle Unterstützung des CD-Labors für Sedimentforschung und -management durch das Bundesministerium für Digitalisierung und Wirtschaftsstandort und die Nationalstiftung für Forschung, Technologie und Entwicklung. Außerdem bedanken wir uns bei den MitarbeiterInnen des Instituts für Wasserbau, Hydraulik und Fließgewässerforschung an der Universität für Bodenkultur Wien für ihren Einsatz für das CD-Labor.

Open Access Dieser Artikel wird unter der Creative Commons Namensnennung 4.0 International Lizenz (http:// creativecommons.org/licenses/by/4. $0 /$ deed.de) veröffentlicht, welche die Nutzung, Vervielfältigung, Bearbeitung, Verbreitung und Wiedergabe in jeglichem Medium und Format erlaubt, sofern Sie den/die ursprünglichen $\mathrm{Au}$ tor(en) und die Quelle ordnungsgemäß nennen, einen Link zur Creative Commons Lizenz beifügen und angeben, ob Änderungen vorgenommen wurden.

Hinweis des Verlags Der Verlag bleibt in Hinblick auf geografische Zuordnungen und Gebietsbezeichnungen in veröffentlichten Karten und Institutsadressen neutral.

\section{Literatur}

Badura, H. (2018): Salzachkraftwerk Gries Konzeption und Bau, Tagungsband Wasserbausymposium Graz 2018, Technische Universitä Graz, Institut für Wasserbau und Wasserwirtschaft, S 305-311

BMLFUW (2014): SED_AT Feststoffhaushalt, Sedimenttransport und Flussmorphologie im Rahmen des Nationalen Gewässerbewirtschaftungsplans. Bundesministerium für Land- und Forstwirtschaft, Umwelt und Wasserwirtschaft, Wien.
BMLFUW (2015a): Nationaler Gewässerbewirtschaftungsplan 2015. Bundesministerium fü Land- und Forstwirtschaft, Umwelt und Wasserwirtschaft, Wien.

BMLFUW (2015b): Task Force Donau: Feinsedimentmanagement an der österreichischen Donau. Endbericht. Bundesministerium für Land- und Forstwirtschaft, Umwelt und Wasserwirtschaft, Wien.

Habersack, H., Blamauer, B., Villwoc,K H., Prenner, D., Hauer, C. (2014): SED AT - Fest- stoffhaushalt, Sedimenttransport und Flussmorphologie im Rahmen des Nationalen Gewässerbewirtschaftungsplans. Österr Wasser- und Abfallw (2014) 66: 327. https://doi.org/10.1007/ s00506-014-0176-9.

Hauer, C., Wagner, B., Aigner, J., Holzapfel, P., Flödl, P., Liedermann, M., Tritthart, M., Sindelar, C., Pulg, U., Klösch, M., Haimann, M., Donnum, B. O., Stickler, M., Habersack, H. (2018): State of the art, shortcomings and future challenges for a sustainable sediment manage- 


\section{Originalarbeit}

ment in hydropower: A review. Renewable and Sustainable Energy Reviews 98 (2018), 40-55. https://doi.org/10.1016/j.rser.2018.08.031

Klasz, G., Reckendorfer, W., Gabriel, H., Baum-

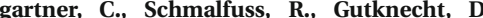

(2014): Natural levee formation along a large and regulated river: The Danube in the National Park Donau-Auen, Austria. Geomorphology 215, 20-33

Sass, O., Harb, G., Stangl, J., Schneider, J. (2015):

ClimCatch-Impact of Climate Change on the sediment yield of small catchments-Final Re- port. 4th Call of the Austrian Climate Research Programme.

Schleiss, A., Oehy, C. (2002): Verlandung von Stauseen und Nachhaltigkeit, In: wasser, energie, luft 94, Heft 7/8, 227-234 